

\title{
Risk bubbles and market instability
}

\author{
Matteo Marsili ${ }^{a, 1}$ and Giacomo Raffaelli ${ }^{\mathrm{b}}$ \\ ${ }^{a}$ Abdus Salam International Centre for Theoretical Physics, Strada Costiera 11, \\ Trieste, Italy \\ ${ }^{\mathrm{b}}$ Dip.to Fisica, Univ. Roma "La Sapienza", P.le A. Moro, Roma, Italy
}

\begin{abstract}
We discuss a simple model of correlated assets capturing the feedback effects induced by portfolio investment in the covariance dynamics. This model predicts an instability when the volume of investment exceeds a critical value. Close to the critical point the model exhibits dynamical correlations very similar to those observed in real markets. Maximum likelihood estimates of the model's parameter for empirical data indeed confirms this conclusion. We show that this picture is confirmed by the empirical analysis for different choices of the time horizon.
\end{abstract}

Globalization and the informatics revolution have expanded the reach and density of financial interactions to an unprecedented extent. At the same time, the complexity of financial instruments has increased in complexity at a much faster pace than our understanding of their consequences on the collective behavior. Somewhat at odds with this, most financial instruments are designed under the assumption that market prices are exogenous processes, i.e. that individuals have no impact at all on the market. Such a price taking assumption might be a good approximation for the individual investor. But, if a particular strategy is used by many traders it will likely have a sizeable impact on the market.

Actually, the Minority Game shows that the global behavior of interacting systems such as a financial markets may change dramatically by the addition of a single agent (see 4.5.3 [1]). It also shows that the properties of financial markets would drastically change if agents accounted, even approximately, for their impact on it (see 5.2.3 in [1]). This occurs because, in the schematic picture of Minority Games, markets have a tendency to react to trading strategies in such a way as to reduce their profitability: The more speculators enter the market, the less this is predictable and the more its state is susceptible to perturbations. The point where the market becomes information-efficient is also

$\overline{1}$ Corresponding author. E-mail: marsili@ictp.it

Preprint submitted to Elsevier Science 12 January 2006 
the point where its susceptibility diverges, implying that the market reacts with a finite response even to infinitesimal perturbations. Interestingly, close to this point Minority Game models exhibit anomalous fluctuations phenomena [2] which bear some resemblance with the stylized facts observed in real markets[3].

We have recently shown [4] that this picture extends, qualitatively, to multiasset markets described by a very simple model. Considerations about risk are essential in this extension ${ }^{2}$. One of the key functions of financial markets is indeed that of allowing companies to "trade" their risk for return, by spreading it across financial investors. Investors on their side, diversify (i.e. spread) their strategies across stocks so as to minimize risk, as postulated by portfolio optimization theory [5]. Ref. [4] discusses how the market is affected, when many agents spread their risk on the market following portfolio theory.

From the point of view of risk, the key quantity of interest is the covariance matrix of asset returns. Much attention has been recently put into the characterization of the statistical properties of the covariance matrix $\hat{C}$ (at the daily scale in most cases): The key findings are that

- The bulk of the eigenvalue distribution of $\hat{C}$ is dominated by noise and it is well described by random matrix theory [6].

- The largest eigenvalue $\Lambda$ - the so-called market mode - is very well separated from the other eigenvalues.

- The few large eigenvalues smaller than $\Lambda$, which leak out of the noise background contain significant economic information. Indeed the taxonomy of assets built [7-10] from financial correlations alone bears remarkable similarity with a classification in economic sectors.

- market correlations also exhibit highly non-trivial dynamics: correlations "build up" as the time scale on which returns are measured increases, and they saturate for returns on the scale of some days [11,12]. Furthermore, financial correlations are persistent over time [13] and they follow recurrent patterns $[10]$.

Fig. 1a shows the time dependence of the largest eigenvalue of (the exponentially averaged) correlation matrix of daily returns for Toronto Stock Exchange [14]. Similar behaviour has been reported earlier [15] for different markets. A closer look [4] at the data in Fig. 1 shows that fluctuations in the largest eigenvalue $\Lambda(t+1)-\Lambda(t)$ are broadly distributed, suggesting that Fig. 1a can hardly be explained entirely as the effect of few exogenous shocks.

Ref. [4] formulates the hypothesis that such non-trivial dynamics arises as a

$\overline{2}$ Most models of single asset markets focus on speculation and expected return. Their generalization to many assets induces no nontrivial dynamics of correlation across assets. 
consequence of the internal market dynamics. The efficiency of portfolio optimization depends on the cross correlations among the stocks the financial market is composed of. But if such optimal portfolio strategies are used by many investors, this will have a sizeable impact on the market. This affects not only price dynamics, but also the very correlations on which these strategies depend. Hence financial correlations enter into a feedback loop because they determine in part those trading strategies which contribute to the price dynamics, i.e. to the financial correlations themselves.

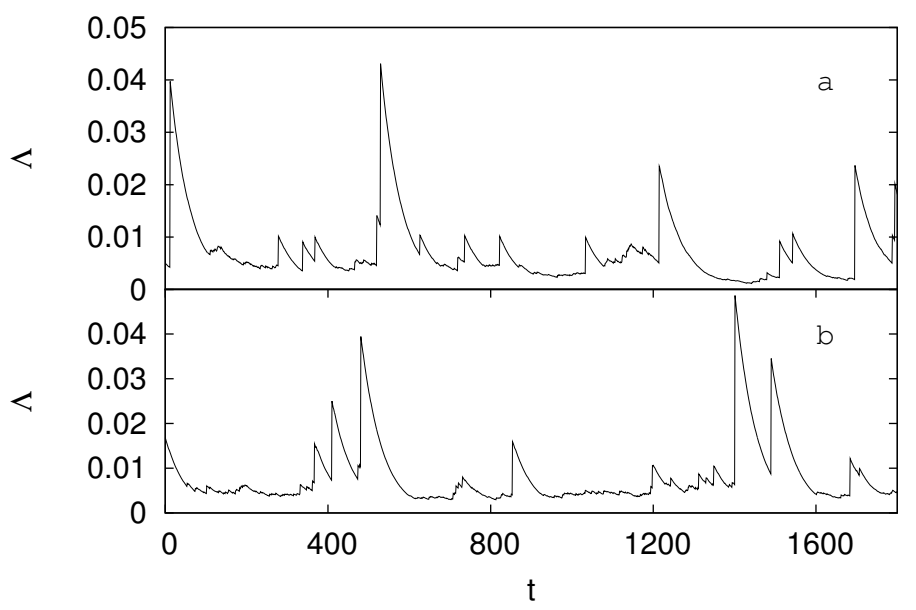

Fig. 1. Maximum eigenvalue of the correlation matrix as a function of time for $\tau=50$. a) Toronto Stock exchange [14]. Here the correlation matrix is obtained using Eqs.(3) and (4) with $\left|\delta x_{t}\right\rangle$ taken from historical data. b) simulation of Eq.(1) with $N=20, R=10^{-2}, B=10^{-6}, \Delta=0.04, \epsilon=10^{-1}, W=1.4$. Components of $|b\rangle$ were generated uniformly in the interval $\left[0,2 \cdot 10^{-3}\right]$, resulting in $W^{*} \approx 1.41$.

The above chain of considerations is captured by a simple phenomenological model [4] aimed at reproducing in a simple yet plausible way the interaction among assets, and the resulting correlations induced by portfolio investment. We consider a market of $N$ assets and we denote by $\left|x_{t}\right\rangle$ the vector of logprices[16] in day $t$. This undergoes the dynamics

$$
\left|x_{t+1}\right\rangle=\left|x_{t}\right\rangle+\left|\beta_{t}\right\rangle+\xi_{t}\left|z_{t}\right\rangle
$$

where $\left|\beta_{t}\right\rangle$ is the vector of bare returns, i.e. it describes the fundamental economic processes which drive the prices. This is assumed to be a Gaussian random vector with $E\left[\left|\beta_{t}\right\rangle\right]=|b\rangle, E\left[\left|\beta_{t}\right\rangle\left\langle\beta_{t^{\prime}}\right|\right]=|b\rangle\langle b|+\hat{B} \delta_{t, t^{\prime}}$, where $|b\rangle$ and $\hat{B}$ are parameters describing the "bare" economic correlations. The last term in Eq. (1) describes the component of trading activity along Markovitz's optimal portfolio. $\xi_{t}$ is an independent Gaussian variable with mean $\epsilon$ and variance $\Delta$ and $\left|z_{t}\right\rangle$ is the optimal portfolio with fixed return $R$ and total wealth $\langle z \mid 1\rangle=W$. In other words, $\left|z_{t}\right\rangle$ is the solution of 


$$
\min _{|z\rangle, \nu, \sigma}\left[\frac{1}{2}\left\langle z\left|\hat{C}_{t}\right| z\right\rangle-\nu\left(\left\langle z \mid r_{t}\right\rangle-R\right)-\sigma(\langle z \mid 1\rangle-W)\right]
$$

where $\hat{C}_{t}$ is the correlation matrix at time $t$. Both the expected returns $\left|r_{t}\right\rangle$ and the correlation matrix $\hat{C}_{t}$, which enter Eq. (2), are computed from historical data over a characteristic time $\tau$ :

$$
\begin{aligned}
\left|r_{t}\right\rangle & =\left(e^{\frac{1}{\tau}}-1\right) \sum_{t^{\prime}<t} e^{-\frac{t-t^{\prime}}{\tau}}\left|\delta x_{t}\right\rangle \\
\hat{C}_{t} & =\left(e^{\frac{1}{\tau}}-1\right) \sum_{t^{\prime}<t} e^{-\frac{t-t^{\prime}}{\tau}}\left|\delta x_{t}\right\rangle\left\langle\delta x_{t}|-| r_{t}\right\rangle\left\langle r_{t}\right|
\end{aligned}
$$

where $\left|\delta x_{t}\right\rangle \equiv\left|x_{t}\right\rangle-\left|x_{t}-1\right\rangle$. This makes the set of equations above a selfcontained dynamical stochastic system. In a nutshell, it describes in a simple way how the economic bare correlated fluctuations $\left|\beta_{t}\right\rangle$ are dressed by financial investment. Note that:

- the optimal portfolio is usually computed from the expected covariance and returns. The model assumes that one can use historical covariance and returns as a proxy for expected ones

- traders have different time horizons in the market whereas the model assumes that all investors have the same trading horizon $\tau$

- a simple linear impact function for the price dynamics is assumed

- a dependence on $\left|\delta z_{t}\right\rangle=\left|z_{t}\right\rangle-\left|z_{t-1}\right\rangle$ can also be envisaged. Indeed, one may think that Eq. (1) contains just the first term in an expansion in time derivatives of $\left|z_{t}\right\rangle$. The rationale for neglecting these terms is that they are less relevant with respect to the $\left|z_{t}\right\rangle$ term, as far as the low frequency behavior of the model is concerned

- the structure of economic correlations is neglected, i.e. $B_{i, j}=B \delta_{i, j}$. The idea behind this is that economic correlations contribute to eigenvalues which are much smaller than the market mode, and hence, to a first approximation are indistinguishable from the noise band. At any rate, economic correlations are expected to change over time scales much longer than those over which the market mode fluctuates. This means that it is unlikely that they contribute in any appreciable way to the dynamics observed in Fig. 1.

- one can consider several component $\left|z_{t}^{k}\right\rangle$ of portfolio investors, each with a different parameters $R^{k}, \epsilon^{k}$ and $\Delta^{k}$. This model can be reduced to the one above, with effective parameters $\tilde{R}, \tilde{\epsilon}$ and $\tilde{\Delta}$

- $\left|z_{t}\right\rangle$ is a quantity, not a percentage as in portfolio theory [5].

Numerical simulations of the model show a very interesting behaviour. In Fig $1 \mathrm{~b}$ we plot the temporal evolution of the maximum eigenvalue $\Lambda$ of the correlation matrix for a particular choice of parameters. The dynamics is highly non-trivial, with the appearance of instabilities resembling those observed in real markets (Fig. 1a). In addition, the statistics of the day-to-day differences 
in $\Lambda$ show a clear power-law behaviour, again very similar to the one we get for real markets.

In the limit $\tau \rightarrow \infty$, where correlations and returns are computed averaging over an infinite time period, the model can be solved analytically [4]. One finds a singular behavior of $\Lambda$ when the volume $W$ of investment in portfolio strategies reaches a critical value $W^{*}$. Indeed as $W \rightarrow W^{*}$ the solution develops a singularity with infinite slope $\frac{\partial \Lambda}{\partial W} \rightarrow \infty$. This is reminiscent of the divergence of susceptibility $\chi$ close to a phase transition, signalling that the response $\delta \Lambda=\chi \delta W$ to a small perturbation $\delta W$ diverges as $W \rightarrow W^{*}$. A static solution ceases to exist in the $\tau \rightarrow \infty$ limit for $W>W^{*}$. This can be seen from a simple geometric argument: For $\epsilon=0$ the two constraints $\langle z \mid 1\rangle=W$ and $\langle z \mid r\rangle=R$ are hyper-planes in the $|z\rangle$ space, hence a solution exists for all $W$ and $R$. With $\epsilon \neq 0$, the constraint $\langle z \mid r\rangle=R$ becomes a hyper-sphere hence, solutions are possible only for $W<W^{*}$.

Numerical simulations show that $W^{*}$ represents a threshold to the amount of wealth that can be invested by risk-minimization into a market without destabilizing it. This is clearly seen in Fig. 2 where we report $\Lambda_{t}$ as a function of time for values of $W$ approaching $W^{*}$. Within the picture offered by the this simple model, Fig. 1 suggests that real markets might be close to the critical point $W^{*}$. This has been checked [4] by fitting real market data [14] with a maximum likelihood method. The whole method was checked to reproduce the correct parameters on synthetic data set generated from the model itself.

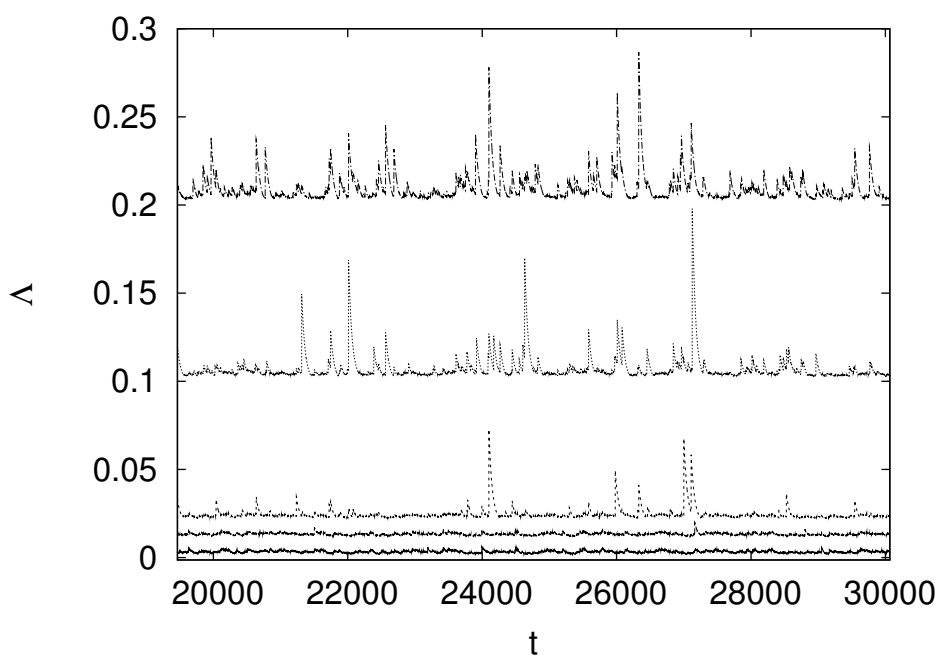

Fig. 2. Maximum eigenvalue of $\hat{C}$ as a function of time for different values of $W$ $(W=1.2,1.32,1.42$ and 1.48 from bottom to top) and the same parameters as in Fig. $1\left(W^{*} \approx 1.42\right)$. Curves are shifted upwards for clarity.

Here we focus on the stability of the results as a function of the time scales used. Indeed, Ref. [4] assumes that the parameters of the model vary slowly on time scales of order $\tau$. We repeated the fitting procedure for different values of 
$\tau$ on different time windows of $T=500$ days. Fig. 3 shows the results for the Dow Jones and the Toronto stock exchange indices. Each window is shifted by 50 days with respect to the previous one, so the data covers approximately 4 years (in order to compute the initial condition for $\hat{C}_{t}$, we used data for 1000 more days). In this time period (1997-2005), no major crashes or structural changes occurred in these markets. The same cannot be said of European markets, because of the introduction of the Euro.

The main figure shows that parameters exhibit some fluctuations but are roughly constant over a long time horizon (the longer time series of the Dow Jones actually displays a very slow convergence to $W^{*}$ in time). The parameters are also stable with respect to changes in $\tau$. The inset shows the location of the fitted parameters with respect to critical point. Results for the same market cluster in the same region for different values of $\tau$.

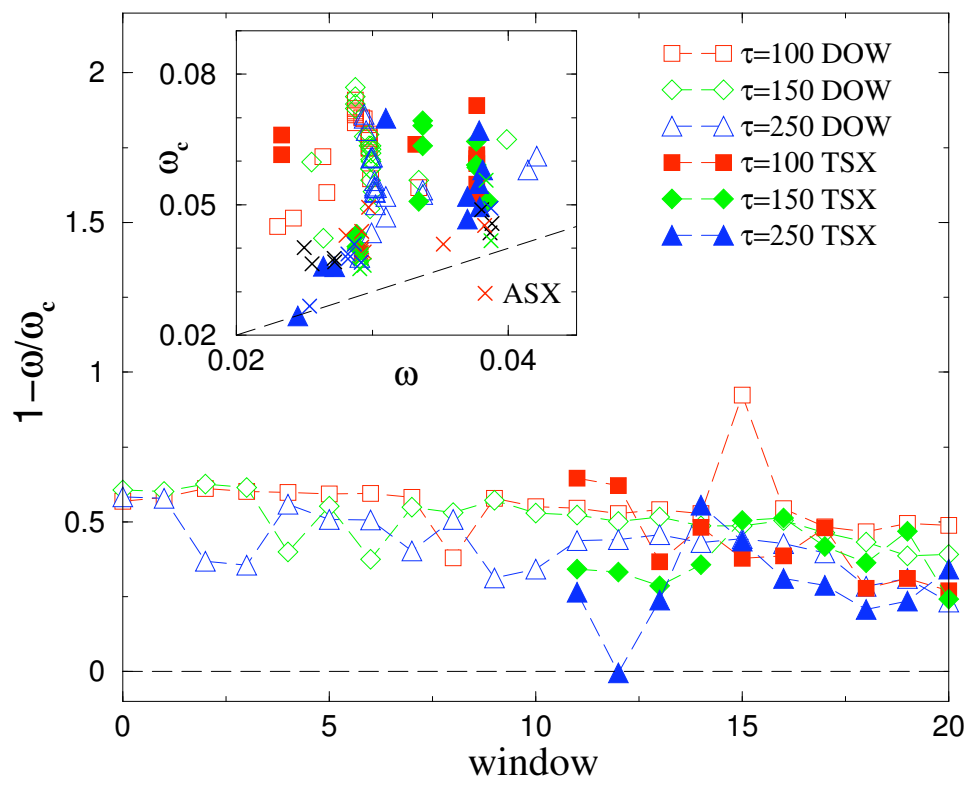

Fig. 3. Fitted values of the parameters $\omega=\epsilon W$ and $\omega_{c}=\epsilon W^{*}$ for successive time windows of $T=500$ points $\tau=100,150$ and 250 and different markets (DJ, TSX, ASX). The main figure shows the distance from the critical point as a function of time. The time window is shifted by 50 days for each point. Inset: $\omega_{c}$ vs $\omega$ for the same data sets.

Our model is very stylized and it fails to capture many important aspects. For example, it is undeniable that external factors and global events have an effect on financial markets. Still it reproduces key empirical features of real financial markets thus suggesting that it captures essential dynamical features of markets. This suggests that the impact of optimal portfolio investment is a potential cause of instability. Non-stationary correlations close to the instability can be considered as an additional component of risk resulting from the enhanced susceptibility of the market. Such "market impact" risk arises because investment according to an optimal portfolio strategy changes 
the structure of correlations with which that strategy was computed. This component of risk diverges as the market approaches the critical point $W^{*}$, thus discouraging further investment. This provides a simple rationale for why markets "self-organize" close to the critical point, which is somewhat reminiscent of the picture which Minority Games [1] provide of single asset markets. The idea is that the market attracts investors as long as it provides efficient ways of diversifying risk. This increases the volume $(W)$ of investment up to the point where the risk measure changes thus turning optimal portfolio strategies into sub-optimal ones.

This is a further case where, when the density of interactions increases, the system approaches a critical state where individual actions might propagate through the system in an unpredictable manner [17]. In such systems, actions might be so remotely related to their ultimate consequences to make even the most sophisticated optimizing behavior or design chimerical.

\section{References}

[1] Challet D., Marsili M. and Zhang Y-C. Minority Games. Interacting agents in financial markets Oxford University Press (Oxford, 2004)

[2] Challet D. and Marsili M. Phys. Rev. E 68 (3) 036132 (2003)

[3] J.-P. Bouchaud and M. Potters Theory of financial risk and derivative pricing: from statistical physics to risk management (Cambridge University Press, Cambridge, 2003); R. Mantegna and E. Stanley, Introduction to Econophysics (Cambridge University Press, 1999).

[4] G. Raffaelli and M. Marsili, eprint physics/0508159.

[5] Elton E.J. and Gruber M.J., Modern Portfolio theory and investment analysis (J. Wiley \& sons, New York, 1995).

[6] Laloux L, Cizeau P, Bouchaud JP, Potters M Phys. Rev. Lett. 83 (7) 14671470 (1999) and Plerou V, Gopikrishnan P, Rosenow B, Amaral LAN, Stanley HE Phys. Rev. Lett. 83 (7) 1471-1474 (1999)

[7] Bonanno G, Caldarelli G, Lillo F, Mantegna RN, Phys. Rev. E 68 4, 046130 (2003)

[8] Plerou V, Gopikrishnan P, Rosenow B, Amaral N.A.N. and Stanley H.E., Phys. Rev. Lett. 83 (7) 1471-1474 (1999).

[9] Giada L. and Marsili M. Phys. Rev. E 63 (6) 061101 (2001)

[10] Marsili M. Quant. Fin. 2 297-302 (2002)

[11] Kwapien J, Drodz S. and Speth J, Phys. A 330 (3-4) 605-621 (2003) 
[12] Potters M. , Bouchaud J.P. and Laloux L., cond-mat/0507111

[13] Onnela JP, Chakraborti A, Kaski K, Kertesz J, Kanto A, Phys. Rev. E 68 (5) $056110(2003)$

[14] Data was taken form finance.yahoo.com in the time period June $16^{\text {th }} 1997$ to May $25^{\text {th }} 2005$ for all assets except for the Dow Jones, for which we used May $2^{\text {nd }} 1995$ to May $23^{\text {rd }} 2005$. Correlations were measured on the set of assets composing the index at the final date.

[15] Drodz S, Kwapien J, Grummer F, Ruf F and Speth J, Phys. A 299 (1-2), 144-153 (2001) and Drodz S, Grummer F, Ruf F and Speth J, Phys. A 294 (1-2), 226-234 (2001).

[16] We use Bra-ket notation: $|x\rangle$ should be considered as a column vector, whereas $\langle x|$ is a row vector. Hence $\langle x \mid y\rangle$ is the scalar product and $|x\rangle\langle y|$ is the direct product, i.e. the matrix with entries $a_{i, j}=x_{i} y_{j}$.

[17] G. Bianconi and M. Marsili, Phys. Rev. E 70, 035105 (2004). 


\section{List of other working papers:}

\section{6}

1. Roman Kozhan, Multiple Priors and No-Transaction Region, WP06-24

2. Martin Ellison, Lucio Sarno and Jouko Vilmunen, Caution and Activism? Monetary Policy Strategies in an Open Economy, WP06-23

3. Matteo Marsili and Giacomo Raffaelli, Risk bubbles and market instability, WP06-22

4. Mark Salmon and Christoph Schleicher, Pricing Multivariate Currency Options with Copulas, WP06-21

5. Thomas Lux and Taisei Kaizoji, Forecasting Volatility and Volume in the Tokyo Stock Market: Long Memory, Fractality and Regime Switching, WP06-20

6. Thomas Lux, The Markov-Switching Multifractal Model of Asset Returns: GMM Estimation and Linear Forecasting of Volatility, WP06-19

7. Peter Heemeijer, Cars Hommes, Joep Sonnemans and Jan Tuinstra, Price Stability and Volatility in Markets with Positive and Negative Expectations Feedback: An Experimental Investigation, WP06-18

8. Giacomo Raffaelli and Matteo Marsili, Dynamic instability in a phenomenological model of correlated assets, WP06-17

9. Ginestra Bianconi and Matteo Marsili, Effects of degree correlations on the loop structure of scale free networks, WP06-16

10. Pietro Dindo and Jan Tuinstra, A Behavioral Model for Participation Games with Negative Feedback, WP06-15

11. Ceek Diks and Florian Wagener, A weak bifucation theory for discrete time stochastic dynamical systems, WP06-14

12. Markus Demary, Transaction Taxes, Traders' Behavior and Exchange Rate Risks, WP06-13

13. Andrea De Martino and Matteo Marsili, Statistical mechanics of socio-economic systems with heterogeneous agents, WP06-12

14. William Brock, Cars Hommes and Florian Wagener, More hedging instruments may destabilize markets, WP06-11

15. Ginwestra Bianconi and Roberto Mulet, On the flexibility of complex systems, WP06-10

16. Ginwestra Bianconi and Matteo Marsili, Effect of degree correlations on the loop structure of scale-free networks, WP06-09

17. Ginwestra Bianconi, Tobias Galla and Matteo Marsili, Effects of Tobin Taxes in Minority Game Markets, WP06-08

18. Ginwestra Bianconi, Andrea De Martino, Felipe Ferreira and Matteo Marsili, Multi-asset minority games, WP06-07

19. Ba Chu, John Knight and Stephen Satchell, Optimal Investment and Asymmetric Risk for a Large Portfolio: A Large Deviations Approach, WP06-06

20. Ba Chu and Soosung Hwang, The Asymptotic Properties of AR(1) Process with the Occasionally Changing AR Coefficient, WP06-05

21. Ba Chu and Soosung Hwang, An Asymptotics of Stationary and Nonstationary AR(1) Processes with Multiple Structural Breaks in Mean, WP06-04

22. Ba Chu, Optimal Long Term Investment in a Jump Diffusion Setting: A Large Deviation Approach, WP06-03

23. Mikhail Anufriev and Gulio Bottazzi, Price and Wealth Dynamics in a Speculative Market with Generic Procedurally Rational Traders, WP06-02

24. Simonae Alfarano, Thomas Lux and Florian Wagner, Empirical Validation of Stochastic Models of Interacting Agents: A "Maximally Skewed" Noise Trader Model?, WP06-01

\section{5}

1. Shaun Bond and Soosung Hwang, Smoothing, Nonsynchronous Appraisal and CrossSectional Aggreagation in Real Estate Price Indices, WP05-17 
2. Mark Salmon, Gordon Gemmill and Soosung Hwang, Performance Measurement with Loss Aversion, WP05-16

3. Philippe Curty and Matteo Marsili, Phase coexistence in a forecasting game, WP05-15

4. Matthew Hurd, Mark Salmon and Christoph Schleicher, Using Copulas to Construct Bivariate Foreign Exchange Distributions with an Application to the Sterling Exchange Rate Index (Revised), WP05-14

5. Lucio Sarno, Daniel Thornton and Giorgio Valente, The Empirical Failure of the Expectations Hypothesis of the Term Structure of Bond Yields, WP05-13

6. Lucio Sarno, Ashoka Mody and Mark Taylor, A Cross-Country Financial Accelorator: Evidence from North America and Europe, WP05-12

7. Lucio Sarno, Towards a Solution to the Puzzles in Exchange Rate Economics: Where Do We Stand?, WP05-11

8. James Hodder and Jens Carsten Jackwerth, Incentive Contracts and Hedge Fund Management, WP05-10

9. James Hodder and Jens Carsten Jackwerth, Employee Stock Options: Much More Valuable Than You Thought, WP05-09

10. Gordon Gemmill, Soosung Hwang and Mark Salmon, Performance Measurement with Loss Aversion, WP05-08

11. George Constantinides, Jens Carsten Jackwerth and Stylianos Perrakis, Mispricing of S\&P 500 Index Options, WP05-07

12. Elisa Luciano and Wim Schoutens, A Multivariate Jump-Driven Financial Asset Model, WP0506

13. Cees Diks and Florian Wagener, Equivalence and bifurcations of finite order stochastic processes, WP05-05

14. Devraj Basu and Alexander Stremme, CAY Revisited: Can Optimal Scaling Resurrect the (C)CAPM?, WP05-04

15. Ginwestra Bianconi and Matteo Marsili, Emergence of large cliques in random scale-free networks, WP05-03

16. Simone Alfarano, Thomas Lux and Friedrich Wagner, Time-Variation of Higher Moments in a Financial Market with Heterogeneous Agents: An Analytical Approach, WP05-02

17. Abhay Abhayankar, Devraj Basu and Alexander Stremme, Portfolio Efficiency and Discount Factor Bounds with Conditioning Information: A Unified Approach, WP05-01

\section{4}

1. Xiaohong Chen, Yanqin Fan and Andrew Patton, Simple Tests for Models of Dependence Between Multiple Financial Time Series, with Applications to U.S. Equity Returns and Exchange Rates, WP04-19

2. Valentina Corradi and Walter Distaso, Testing for One-Factor Models versus Stochastic Volatility Models, WP04-18

3. Valentina Corradi and Walter Distaso, Estimating and Testing Sochastic Volatility Models using Realized Measures, WP04-17

4. Valentina Corradi and Norman Swanson, Predictive Density Accuracy Tests, WP04-16

5. Roel Oomen, Properties of Bias Corrected Realized Variance Under Alternative Sampling Schemes, WP04-15

6. Roel Oomen, Properties of Realized Variance for a Pure Jump Process: Calendar Time Sampling versus Business Time Sampling, WP04-14

7. Richard Clarida, Lucio Sarno, Mark Taylor and Giorgio Valente, The Role of Asymmetries and Regime Shifts in the Term Structure of Interest Rates, WP04-13

8. Lucio Sarno, Daniel Thornton and Giorgio Valente, Federal Funds Rate Prediction, WP04-12

9. Lucio Sarno and Giorgio Valente, Modeling and Forecasting Stock Returns: Exploiting the Futures Market, Regime Shifts and International Spillovers, WP04-11

10. Lucio Sarno and Giorgio Valente, Empirical Exchange Rate Models and Currency Risk: Some Evidence from Density Forecasts, WP04-10

11. Ilias Tsiakas, Periodic Stochastic Volatility and Fat Tails, WP04-09

12. Ilias Tsiakas, Is Seasonal Heteroscedasticity Real? An International Perspective, WP04-08

13. Damin Challet, Andrea De Martino, Matteo Marsili and Isaac Castillo, Minority games with finite score memory, WP04-07

14. Basel Awartani, Valentina Corradi and Walter Distaso, Testing and Modelling Market Microstructure Effects with an Application to the Dow Jones Industrial Average, WP04-06 
15. Andrew Patton and Allan Timmermann, Properties of Optimal Forecasts under Asymmetric Loss and Nonlinearity, WP04-05

16. Andrew Patton, Modelling Asymmetric Exchange Rate Dependence, WP04-04

17. Alessio Sancetta, Decoupling and Convergence to Independence with Applications to Functional Limit Theorems, WP04-03

18. Alessio Sancetta, Copula Based Monte Carlo Integration in Financial Problems, WP04-02

19. Abhay Abhayankar, Lucio Sarno and Giorgio Valente, Exchange Rates and Fundamentals: Evidence on the Economic Value of Predictability, WP04-01

\section{2}

1. Paolo Zaffaroni, Gaussian inference on Certain Long-Range Dependent Volatility Models, WP02-12

2. Paolo Zaffaroni, Aggregation and Memory of Models of Changing Volatility, WP02-11

3. Jerry Coakley, Ana-Maria Fuertes and Andrew Wood, Reinterpreting the Real Exchange Rate - Yield Diffential Nexus, WP02-10

4. Gordon Gemmill and Dylan Thomas, Noise Training, Costly Arbitrage and Asset Prices: evidence from closed-end funds, WP02-09

5. Gordon Gemmill, Testing Merton's Model for Credit Spreads on Zero-Coupon Bonds, WP0208

6. George Christodoulakis and Steve Satchell, On th Evolution of Global Style Factors in the MSCI Universe of Assets, WP02-07

7. George Christodoulakis, Sharp Style Analysis in the MSCI Sector Portfolios: A Monte Caro Integration Approach, WP02-06

8. George Christodoulakis, Generating Composite Volatility Forecasts with Random Factor Betas, WP02-05

9. Claudia Riveiro and Nick Webber, Valuing Path Dependent Options in the Variance-Gamma Model by Monte Carlo with a Gamma Bridge, WP02-04

10. Christian Pedersen and Soosung Hwang, On Empirical Risk Measurement with Asymmetric Returns Data, WP02-03

11. Roy Batchelor and Ismail Orgakcioglu, Event-related GARCH: the impact of stock dividends in Turkey, WP02-02

12. George Albanis and Roy Batchelor, Combining Heterogeneous Classifiers for Stock Selection, WP02-01

\section{1}

1. Soosung Hwang and Steve Satchell, GARCH Model with Cross-sectional Volatility; GARCHX Models, WP01-16

2. Soosung Hwang and Steve Satchell, Tracking Error: Ex-Ante versus Ex-Post Measures, WP01-15

3. Soosung Hwang and Steve Satchell, The Asset Allocation Decision in a Loss Aversion World, WP01-14

4. Soosung Hwang and Mark Salmon, An Analysis of Performance Measures Using Copulae, WP01-13

5. Soosung Hwang and Mark Salmon, A New Measure of Herding and Empirical Evidence, WP01-12

6. Richard Lewin and Steve Satchell, The Derivation of New Model of Equity Duration, WP0111

7. Massimiliano Marcellino and Mark Salmon, Robust Decision Theory and the Lucas Critique, WP01-10

8. Jerry Coakley, Ana-Maria Fuertes and Maria-Teresa Perez, Numerical Issues in Threshold Autoregressive Modelling of Time Series, WP01-09

9. Jerry Coakley, Ana-Maria Fuertes and Ron Smith, Small Sample Properties of Panel Timeseries Estimators with I(1) Errors, WP01-08

10. Jerry Coakley and Ana-Maria Fuertes, The Felsdtein-Horioka Puzzle is Not as Bad as You Think, WP01-07

11. Jerry Coakley and Ana-Maria Fuertes, Rethinking the Forward Premium Puzzle in a Nonlinear Framework, WP01-06

12. George Christodoulakis, Co-Volatility and Correlation Clustering: A Multivariate Correlated ARCH Framework, WP01-05 
13. Frank Critchley, Paul Marriott and Mark Salmon, On Preferred Point Geometry in Statistics, WP01-04

14. Eric Bouyé and Nicolas Gaussel and Mark Salmon, Investigating Dynamic Dependence Using Copulae, WP01-03

15. Eric Bouyé, Multivariate Extremes at Work for Portfolio Risk Measurement, WP01-02

16. Erick Bouyé, Vado Durrleman, Ashkan Nikeghbali, Gael Riboulet and Thierry Roncalli, Copulas: an Open Field for Risk Management, WP01-01

\section{0}

1. Soosung Hwang and Steve Satchell, Valuing Information Using Utility Functions, WP00-06

2. Soosung Hwang, Properties of Cross-sectional Volatility, WP00-05

3. Soosung Hwang and Steve Satchell, Calculating the Miss-specification in Beta from Using a Proxy for the Market Portfolio, WP00-04

4. Laun Middleton and Stephen Satchell, Deriving the APT when the Number of Factors is Unknown, WP00-03

5. George A. Christodoulakis and Steve Satchell, Evolving Systems of Financial Returns: AutoRegressive Conditional Beta, WP00-02

6. Christian S. Pedersen and Stephen Satchell, Evaluating the Performance of Nearest Neighbour Algorithms when Forecasting US Industry Returns, WP00-01

\section{9}

1. Yin-Wong Cheung, Menzie Chinn and Ian Marsh, How do UK-Based Foreign Exchange Dealers Think Their Market Operates?, WP99-21

2. Soosung Hwang, John Knight and Stephen Satchell, Forecasting Volatility using LINEX Loss Functions, WP99-20

3. Soosung Hwang and Steve Satchell, Improved Testing for the Efficiency of Asset Pricing Theories in Linear Factor Models, WP99-19

4. Soosung Hwang and Stephen Satchell, The Disappearance of Style in the US Equity Market, WP99-18

5. Soosung Hwang and Stephen Satchell, Modelling Emerging Market Risk Premia Using Higher Moments, WP99-17

6. Soosung Hwang and Stephen Satchell, Market Risk and the Concept of Fundamental Volatility: Measuring Volatility Across Asset and Derivative Markets and Testing for the Impact of Derivatives Markets on Financial Markets, WP99-16

7. Soosung Hwang, The Effects of Systematic Sampling and Temporal Aggregation on Discrete Time Long Memory Processes and their Finite Sample Properties, WP99-15

8. Ronald MacDonald and Ian Marsh, Currency Spillovers and Tri-Polarity: a Simultaneous Model of the US Dollar, German Mark and Japanese Yen, WP99-14

9. Robert Hillman, Forecasting Inflation with a Non-linear Output Gap Model, WP99-13

10. Robert Hillman and Mark Salmon, From Market Micro-structure to Macro Fundamentals: is there Predictability in the Dollar-Deutsche Mark Exchange Rate?, WP99-12

11. Renzo Avesani, Giampiero Gallo and Mark Salmon, On the Evolution of Credibility and Flexible Exchange Rate Target Zones, WP99-11

12. Paul Marriott and Mark Salmon, An Introduction to Differential Geometry in Econometrics, WP99-10

13. Mark Dixon, Anthony Ledford and Paul Marriott, Finite Sample Inference for Extreme Value Distributions, WP99-09

14. Ian Marsh and David Power, A Panel-Based Investigation into the Relationship Between Stock Prices and Dividends, WP99-08

15. Ian Marsh, An Analysis of the Performance of European Foreign Exchange Forecasters, WP99-07

16. Frank Critchley, Paul Marriott and Mark Salmon, An Elementary Account of Amari's Expected Geometry, WP99-06

17. Demos Tambakis and Anne-Sophie Van Royen, Bootstrap Predictability of Daily Exchange Rates in ARMA Models, WP99-05

18. Christopher Neely and Paul Weller, Technical Analysis and Central Bank Intervention, WP9904

19. Christopher Neely and Paul Weller, Predictability in International Asset Returns: A Reexamination, WP99-03 
20. Christopher Neely and Paul Weller, Intraday Technical Trading in the Foreign Exchange Market, WP99-02

21. Anthony Hall, Soosung Hwang and Stephen Satchell, Using Bayesian Variable Selection Methods to Choose Style Factors in Global Stock Return Models, WP99-01

\section{8}

1. Soosung Hwang and Stephen Satchell, Implied Volatility Forecasting: A Compaison of Different Procedures Including Fractionally Integrated Models with Applications to UK Equity Options, WP98-05

2. Roy Batchelor and David Peel, Rationality Testing under Asymmetric Loss, WP98-04

3. Roy Batchelor, Forecasting T-Bill Yields: Accuracy versus Profitability, WP98-03

4. Adam Kurpiel and Thierry Roncalli, Option Hedging with Stochastic Volatility, WP98-02

5. Adam Kurpiel and Thierry Roncalli, Hopscotch Methods for Two State Financial Models, WP98-01 